\title{
Humanités numériques et formation innovante en didactique du FLE.
}

Un enjeu pour une mobilité différenciée

Jérémi Sauvage

\section{(2) OpenEdition \\ Journals}

Édition électronique

URL : http://journals.openedition.org/rdlc/7051

DOI : $10.4000 /$ rdlc.7051

ISSN : 1958-5772

Éditeur

ACEDLE

Référence électronique

Jérémi Sauvage, «Humanités numériques et formation innovante en didactique du FLE. 》, Recherches en didactique des langues et des cultures [En ligne], 16-2 | 2019, mis en ligne le 01 septembre 2019 consulté le 10 décembre 2020. URL : http://journals.openedition.org/rdlc/7051 ; DOI : https://doi.org/ $10.4000 /$ rdlc.7051

Ce document a été généré automatiquement le 10 décembre 2020.

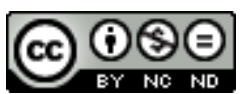

Recherches en didactique des langues et des cultures is licensed under a Creative Commons AttributionNonCommercial-NoDerivatives 4.0 International License 


\title{
Humanités numériques et formation innovante en didactique du FLE.
}

Un enjeu pour une mobilité différenciée

\author{
Jérémi Sauvage
}

\section{Introduction}

1 Cet article se veut une approche générale à propos des enjeux impliqués à la fois dans la numérisation (ou la digitalisation selon les sources) des situations d'enseignementapprentissage mais également dans leurs prises en compte dans les démarches d'innovation pédagogique. Pour ce faire, nous proposons de nous appuyer tout au long de notre réflexion sur le statut du laboratoire de phonétique (et de ses utilisations) en didactique des langues, et en particulier en didactique du FLE. Nous serons donc amenés à (ré-)interroger certains concepts tels que «innovation», «enjeux numériques" mais également à prendre en compte des réalités sociales et sociétales tels que la démocratisation des usages numériques ainsi que le développement des mobilités étudiantes dans le cadre d'études universitaires en langues ou dans d'autres disciplines. Nous pensons qu'il est fondamental de poursuivre le questionnement afin de mieux répondre à des besoins réels.

2 Aussi, nous proposons de garder en perspective l'articulation permanente entre la réflexion didactique et la réflexion pédagogique. Tout un chacun aura pu être témoin d'un décalage possible entre le "discours didactique", par exemple en formation de formateurs, et les «pratiques de classe» s'inscrivant dans une réalité et une concrétude. Nous pouvons ainsi constater que les étudiants de formation initiale comme le master FLE sont souvent déroutés par ce décalage saillant entre ce qui est préconisé en cours et la réalité du terrain de stage. En ce qui nous concerne, nous avons maintes fois fait ce constat, notamment à propos des activités effectives en laboratoire de phonétique dans la classe de FLE. Si le discours professoral se donne pour objectif pertinence et efficacité au travers de situations simples (des cas d'école où les apprenants sont peu nombreux avec tous exactement le même niveau sur les plans 
perceptif et articulatoire), force est de constater que la complexité du terrain ne rend pas compte de la réalité du quotidien de l'enseignant de langue.

3 On peut ainsi constater, pour ce qui est de la didactique de la phonétique, un paradoxe explicable d'un point de vue épistémologique (Sauvage \& Billières, 2019; Sauvage, 2019b; Weber, 2019): il existe peu de formations exhaustives dans le domaine (comparé à d'autres domaines linguistiques comme la grammaire ou la conversation en FLE) et en même temps, on peut noter une forte demande de la part des enseignants pour se former sur les plans théorique et pratique.

\section{Vous avez dit innovation?}

4 Un danger possible dans le présent domaine est notamment celui du galvaudage de termes et de concepts. Ce fut par exemple le cas avec les fameuses "Nouvelles" technologies de l'information et de la communication, qui sont parfois restées nouvelles des années après leur apparition, du plan Informatique pour tous (IPT) lancé en 1985 dans l'Education nationale jusqu'au Nouvelles Technologies de l'Information et de la Communication pour l'Enseignement (NTICE) dans les années 1990, devenue TICE dans les années 2000 (Fourgous, 2010). Pourtant l'apparition de nouvelles ressources et de nouveaux outils redéfinit constamment ce qui peut relever d'une innovation.

5 En nous référant au dictionnaire de langue Le Littré, le terme innovation renvoie à "l'action d'innover » et au "résultat de cette action». Le terme innover renvoie lui à l'action de "changer par esprit et désir de nouveauté ». On peut alors se demander ce que le pédagogue désireux d'innover désire changer et pour quelles raisons. Mais selon le domaine disciplinaire dans lequel on s'inscrit, Cros (2001) insiste sur la polysémie du terme : «Selon la perspective sociologique, «innovation est un changement de règles par celui qui n'en a pas la maîtrise" (Bernoux, 1995); selon la perspective psychologique, c'est la création de nouvelles pratiques fondées sur l'inventivité (Moscovici, 1977) ; selon la perspective managériale et politique, "l' innovation est toute tentative visant consciemment et délibérément à introduire dans le système un changement dans le but d'aménager le système »; selon l'approche des sciences de l'éducation, « innovation, c'est l'introduction délibérée d'un changement spécifique » (Cros, $2001: 21-22$ ).

\section{Innover à partir de l'existant}

6 L'un des objectifs possibles pour le chercheur est, nous semble-t-il, de partir d'une situation existante et d'essayer d'en modifier le contenu. Par exemple, le didacticien pourra avoir à cœur de faire évoluer un curriculum afin d'en adapter le contenu aux réalités sociétales. C'est le constat souvent fait à propos des changements des Programmes et autres Instructions officielles de l'Education nationale. On s'appuie sur ce qui existe et l'on décide de parfaire l'existant en approfondissant telle ou telle idée force, mais toujours dans la même inscription doxique (Sauvage, 2017).

7 Il est également possible de changer le contenu de l'existant et de présenter le même curriculum sous une forme nouvelle, par exemple numérique. Si l'on veut parler ici d'innovation numérique, il nous semble que l'abus de langage est touché du doigt. Téléverser un fichier au format PDF sur une plateforme numérique ne constitue pas en soi une innovation pédagogique (sur le fond) mais une évolution de la présentation du 
texte pédagogique, tout comme le fait de lire un livre en format papier ou sur une liseuse. Par contre, l'innovation ici implique une évolution sur le plan des usages sociaux, ce qui constitue en soi un domaine de réflexion à part entière. L'intérêt dans ce cas est de s'interroger sur les implications sous-jacentes: en quoi le changement de nature des supports de formations conduit à réorganiser cette formation, voire à créer une formation nouvelle s'appuyant pleinement sur ces nouveaux usages sociaux ?

8 Enfin, par voie de conséquence, innover le contenu ou la forme pédagogique d'une formation renvoie immanquablement à une innovation administrative et/ou technologique. Nous menons ainsi une réflexion collective à l'Université Paul-Valéry Montpellier 3 pour créer un diplôme d'université d'études du Français (DUEF) répondant à des besoins nouveaux : enseigner une langue en proposant un dispositif assez souple pour que les étudiants-apprenants puissent suivre des cours en compréhension, en production, à l'oral à l'écrit correspondant à leur niveau réel. Il n'est par exemple pas rare qu'un apprenant ait un niveau de compétence attesté A2 en production orale mais déjà $\mathrm{B} 1$ en compréhension écrite (ou l'inverse). Mais sur un plan purement administratif, l'organisation des évaluations, des attestations de notes et de diplômes, il devient trop complexe de partir de l'organisation existante s'appuyant sur des DUEF complètement indépendants car en conformité avec les niveaux de compétence prescrits par le CECRL (Conseil de l'Europe, 2001 ; 2018). Il convient alors de faire évoluer des procédures administratives. Il devient alors nécessaire de proposer une rupture avec ce qui existe déjà pour inventer des alternatives réellement innovantes.

\section{Innover en rupture avec l'existant}

9 Parce qu'il n'est pas toujours possible de partir de l'existant, la création de nouveaux outils, de nouvelles procédures, s'impose parfois comme une nécessité.

La question administrative est prégnante de ce point de vue. L'organisation générale de notre nouveau DUEF se voulant innovante car beaucoup plus souple et adaptée par rapport à ce qui existe, nous avons pensé le calendrier général en nous appuyant sur certaines réalités organisationnelles d'universités étrangères. Ainsi, les trois temps forts de l'hybridation seront organisés comme suit : un premier séjour à Montpellier en juin-juillet; puis un retour de l'étudiant dans son université d'origine avec un suivi durant toute l'année universitaire ; et enfin un retour en juin-juillet à Montpellier pour valider les examens qui n'ont pas été évaluée en ligne et donc finaliser la formation. Le principal problème est que, en France, une année universitaire sur le plan administratif ne commence qu'en septembre (ni en juin, ni en juillet). Comment peut-on inscrire officiellement des étudiants en dehors du calendrier administratif, notamment pour leur permettre d'accéder aux différents services fournis (en particulier à leur environnement numérique de travail) sans effectuer deux fois la démarche administrative? On le voit ici, l'innovation pédagogique une fois conceptualisée implique une innovation extra-pédagogique. La souplesse nouvelle de la formation conduit à une nécessaire adaptation pour faire évoluer l'organisation administrative de la Direction des études et de la scolarité.

11 Sur le plan pédagogique, notamment en phonétique, créer une formation hybride impliquant des situations d'enseignement-apprentissage distancielles nous conduit à repenser le contenu et l'organisation de cette formation (Charlier, Deschryver \& 
Peraya, 2006). Impossible d'échapper à la création de nouveaux outils pédagogiques, pour éviter notamment de tomber dans une transposition simpliste d'un cours en présentiel. Il s'agit alors de s'appuyer sur les possibilités de la plateforme numérique utilisée et d'imaginer des possibles en cohérence avec les objectifs, notamment ceux évoqués supra à propos des discours didactiques. Ainsi, en phonétique corrective, réfléchir sur l'élaboration de séquences pédagogiques s'appuyant sur un laboratoire de phonétique dématérialisé pour favoriser des apprentissages en autonomie (avec ou sans tutorat - Depover, De Lièvre, Peraya, Quintin \& Jaillet, 2011) et l'adaptation aux difficultés effectives des apprenants s'impose comme une évidence. Cela passe alors par une conceptualisation d'outils, d'activités et d'exercices qui n'existent pas encore car c'est ce que requiert leur transposition à la situation distancielle. Nouveaux outils, nouveaux dispositifs et nouvelles pratiques d'enseignement et d'apprentissage deviennent donc des priorités et des objets de recherche à part entière.

\section{Quels enjeux numériques?} étudiants qui sont souvent plus facile à joindre via Snapchat, Whatsapp ou Facebook plutôt que par des canaux plus académiques et universitaires (Peraya \& Bonfils, 2014). L'étude des usages sociaux grâce à ces nouveaux moyens de communication interindividuels constitue en soi un champ important dans le domaine des humanités numériques (Denouël \& Granjon, 2011; Mangenot \& Soubrié, 2014 : Soubrié, 2014 ; Grassin, 2017) et de la didactique des langues (Grassin, 2016).Un étudiant bénéficie aujourd'hui d'une connectivité permanente et nul doute que ces nouvelles pratiques participent à la construction des personnes sociales et des citoyens de demain (Martin, 2012).

14 Un autre aspect concerne le contenu des formations, par l'entremise de la digitalisation des savoirs et des connaissances d'une part, la digitalisation des formations d'autre part (Develotte, 2010 ; Charnet, 2016; Soubrié, 2016). Tout cela se traduit depuis plusieurs années par de nouveaux formats de formation comme les Formations en ligne ouvertes à tous (FLOT ou MOOC - Oliveri \& Rasse, 2017), les Formations ouvertes à distance (FOAD), etc. (Bernard, 2003).

Un troisième aspect vient compléter les deux premiers précédemment évoqués : la scénarisation des formations pédagogiques (Nissen, 2004 ; 2006 ; 2011 ; Bakki, Oubahssi, George \& Cherkaoui, 2017) et leur gamification (Pomian, 2009 ; Cohard, 2015). Scénariser une formation, c'est entrer de plain-pied dans l'ère numérique de l'apprentissage. Apprendre une langue ou autre chose, c'est suivre un parcours, vivre une série d'enchainements. Dans la société d'aujourd'hui, marquée par des usages quotidiens de médiation numérique, principalement grâce aux smartphones, la ludification permet de dépasser l'ancestrale et désuète (bien qu'étymologique) association travail/souffrance pour aller vers l'apprentissage/plaisir. Il devient alors possible pour l'apprenant de franchir des niveaux, de marquer des points, d'obtenir des bonus au fur et à mesure qu'il progresse dans son parcours d'apprentissage. L'étudiant né autour de l'an 2000 ayant toujours connu des interfaces numériques, il nous semble essentiel de tenir

\section{Recherches en didactique des langues et des cultures, 16-2 | 2019}


compte de ce paramètre pour penser les organisations pédagogiques de demain. Outre l'adaptation nécessaire à une nouvelle génération d'étudiants, il s'agit également de prendre en compte les résultats des travaux s'inscrivant dans le champ de la psychologie des émotions, pour laquelle le pathos et l'éthos sont des facteurs à ne pas écarter dans un contexte d'apprentissage (Sander, 2014).

Enfin, l'apprentissage en autonomie partielle ou totale n'est plus un sujet innovant, que ce soit à propos d'autodidaxie (Tremblay, 1986; André, 1989) ou d'autoformation (Carré, Moisan, Poisson \& Cyrot, 2010), dans la continuité des fameuses méthodes Assimil ou Langues pour tous du $20^{\mathrm{e}}$ siècle. Mais la démocratisation des usages numériques est en lien avec le développement d'une ingénierie commerciale de portails et/ou d'applications pour apprendre une langue étrangère (Nissen, 2007). L'autoformation a alors pris un virage important dans les années 2000 (Barbot, 2000 ; Barbot \& Pugibet, 2002 ; Barbier-Bouvet, 2013), à notre avis parce qu'elle offre ce dispositif d'adaptation à l'apprenant, par exemple au niveau de la gestion du rythme d'apprentissage et de sa progression (Berdal-Masuy, Briet \& Pairon, 2004). Dès lors, la réflexion s'oriente sur certaines spécificités entre les possibilités de cette médiation numérique de plus en plus présente et les enjeux présents dans l'apprentissage d'une langue étrangère (Narcy-Combes, 2005).

\section{Innovations numérique et pédagogique?}

17 A la suite de ce qui a été évoqué supra, nous pouvons nous interroger sur ce qui relève de l'innovation sur les plans numérique et pédagogique. Pour ce faire, il faut donc envisager d'inventer de nouveaux outils numériques et certainement aussi de nouvelles pratiques. Dans cette optique, nous souhaitons lancer une réflexion sur un laboratoire de phonétique entièrement numérique, rattaché à une plateforme numérique d'apprentissage linguistique. En situation distancielle et/ou autodidaxique, l'apprenant doit pouvoir progresser à son rythme, en perception et/ou en articulation, sur des remédiations phonologiques qui sont propres à sa situation personnelle, ce qui induit une prise en compte différenciée sur le plan pédagogique mais également sur celui de la mobilité. Par exemple, l'apprenant chinois aura forcément besoin d'une série de séquences pour travailler l'opposition par le voisement des consonnes occlusives de la langue française $(/ \mathrm{p} / \sim / \mathrm{b} / ; / \mathrm{t} / \sim / \mathrm{d} / ; / \mathrm{k} / \sim / \mathrm{g} /)$, ce qui ne constituera pas un objet d'enseignement-apprentissage pour des apprenants non-chinois.

Un autre aspect, tout aussi important pour renforcer la pertinence pédagogique d'une séance de phonétique consistera à dépasser l'étiquetage artificiel des étudiants en DUEF auxquels on attribue trop facilement de manière simpliste un niveau général A1, A2, B1, etc. Or, sur le plan phonétique, un apprenant reconnu comme ayant un niveau général B2 acquis peut tout à fait avoir besoin d'une leçon ou d'une séquence pédagogique proposées à un débutant niveau A. Nous pensons que la médiatisation numérique, la scénarisation de la formation ainsi que l'organisation technologique d'une plateforme peuvent permettre de répondre de manière précise et pertinente à ce besoin de souplesse. Notons au passage, que la mise en place de ce genre de dispositif pourrait constituer un complément tout aussi pertinent aux formations en présentiel, justifiant l'hybridation nécessaire d'une formation et offrant une voie possible de la prise en compte de l'hétérogénéité existant dans une classe de FLE (en particulier dans un 
Centre universitaire accueillant plusieurs centaines d'étudiants par semestre issus de dizaines de pays différents).

On le voit ici, cette nécessité constante d'inventer, d'innover, à la fois en pratiques numériques et sur le plan pédagogique invite à réfléchir à une innovation didactique. Les pistes de réflexions sont conditionnées par l'organisation et la fluidité de la constitution d'un programme individuel adapté pour un apprenant donné, ce qui inclut un nombre d'exercices et d'activités suffisant pour répondre à différents scénarios possibles : l'un aura plus besoin de passer du temps sur un entrainement pour travailler la perception, l'autre devra avoir la possibilité d'en passer davantage sur des exercices de répétition avec une option d'autodidaxie intégrée pour comparer sa prononciation autant de fois qu'il le souhaite au modèle à atteindre. Par ailleurs, le réinvestissement de ces entrainements mécaniques indispensables devra trouver également sa place dans des activités de conversations authentiques en ligne, ce qui implique, pour l'enseignant, de penser une méthodologie de conversation synchrone afin de mettre en pratique ce qui aura été appris en compréhension et en production orales. Nous préconisons notamment une organisation systémique impliquant des étudiants de Master en didactique des langues qui pourraient, dans le cadre d'un stage, intégrer cette formation hybride en offrant plus que du virtuel et de la décontextualisation (comme des supports ou des textes prétextes) dans le cadre d'une formation de langue : ils pourraient alors concevoir et alimenter une plateforme à destination des étudiants en mobilité, ce qui leur permettrait de mieux saisir les enjeux du travail phonétique.

\section{En guise de conclusion : mobilité différenciée et formation innovante}

20 Un dernier point que nous souhaitons évoquer est la question de la mobilité des étudiants et sa prise en compte dans l'élaboration de formation innovante en langue étrangère. D'emblée, la médiation numérique et l'autoformation apparaissent comme des voies force de proposition pour répondre à ces besoins de formation spécifiques.

Ainsi, Guichon, Koné \& Grassin (2017) problématisent la transition numérique du point de vue de la didactique. Comment construire une réflexion didactique pertinente face à la diversité des pratiques et des acculturations à l'apprentissage d'une langue comme le français, alors même que l'une des ambitions d'une formation innovante est justement de répondre à ce besoin? Le groupement Campus FLE - ADCUEFE, qui réunit la quasitotalité des centres universitaires d'enseignement du FLE en France, a justement pour légitime ambition de répondre à cette question en réfléchissant et en proposant des pistes de réflexion. L'hybridation des formations DUEF et le cadrage de leur organisation devient alors un sujet brulant. Nous avons ainsi pu montrer (Sauvage, Dufour \& Auger, 2017) que les étudiants en mobilité universitaire encadrée (type Erasmus) éprouvaient ce besoin d'accompagnement en langue française en plus du programme de formation disciplinaire suivi. Finalement, il nous incombe d'inventer le futur numérique de la didactique des langues. Par exemple, étant donné les besoins exprimés pour les formations en phonétique, cette réflexion à propos de la conception d'un laboratoire de phonétique entièrement numérisé apparaît comme une priorité pour les prochaines années. Mais attention! Prenons garde de ne pas simplement numériser l'existant (qui reste améliorable - Sauvage \& Billières, 2019). De manière tautologique, innover dans ce domaine implique des propositions innovantes, tant sur 
le fond que sur la forme pour, enfin, atteindre les objectifs fixés par les différents curricula (Certifications linguistiques DELF/DALF), objectifs du CECR (Conseil de l'Europe, 2018). Aujourd'hui, les étudiants en mobilité peuvent ne pas se déplacer géographiquement mais, grâce à la médiation numérique, bénéficier d'une mobilité virtuelle (Sauvage, 2019a).

Nous avons essayé dans cet article de mettre en évidence un certain nombre d'éléments pour proposer une démarcher d'adaptation pédagogique via le numérique en didactique des langues, en questionnant des concepts nous semblant fondamentaux. La question de la transition numérique tout d'abord nous apparaît comme incontournable et mérite à elle seule de réinterroger sur les plans didactique et pédagogique l'enseignement-apprentissage des langues étrangères en général, du FLE en particulier. Etant donné les usages numériques des étudiants actuels et des futurs étudiants, il est indispensable de prendre en compte l'évolution de ces pratiques sociales, langagières et technologiques pour concevoir des formations en phonétique innovantes. Le domaine phonético-phonologique nécessite, comme c'est déjà le cas pour la classe de langue, une réflexion particulière quant à sa transposition dans le cadre d'une interface numérique, à distance et en autonomie. Mais innover d'un point de vue pédagogique invite l'ensemble de la communauté à innover également, sur les plans administratif et technologique. L'objectif général se traduit donc ici par une volonté de penser le futur et d'anticiper certaines pratiques pour s'adapter le plus facilement à des réalités sociales et sociétales et à différencier les formes de mobilité étudiante.

\section{BIBLIOGRAPHIE}

Bakki, A., Oubahssi, L., George, S. \& Cherkaoui, C. (2017). Approche et outils pour assister la scénarisation pédagogique des MOOCs. 8ème Conférence sur les Environnements Informatiques pour l'Apprentissage Humain, Juin 2017, Strasbourg, 185-196.

André, B. (1989). Autonomie et enseignement/Apprentissage des langues étrangères. Paris : DidierHatier.

Barbier-Bouvet, J. F. (2013). Babel à Beaubourg: L'autodidaxie linguistique à la Bpi. Enquête sur la

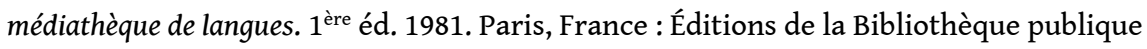
d'information.

Barbot, M. J. (2000). Les auto-apprentissages. Paris : CLE international.

Barbot, M. J., \& Pugibet, V. (2002). Apprentissages des langues et technologies : usages en émergence. Paris : Clé International. Berdal-Masuy, F., Briet, G. \& Pairon, J. (2004). Apprendre seul, à son rythme et encadré. Études de linguistique appliquée, 134(2), 173-190.

Bernoux, Ph. (1995). La sociologie des entreprises. Ed. 2009, Paris : Seuil.

Bertrand, I. (2003). Les dispositifs de FOAD dans les établissements d'enseignement supérieur : transfert ou intégration? Distances et savoirs, vol. 1(1), 61-78. Doi :10.3166/ds.1.61-78. 
Carré, P., Moisan, A., Poisson, D., \& Cyrot, P. (2010). L'autoformation : perspectives de recherche. Paris : Presses universitaires de France.

Charnet, C. (2016). Et si le numérique changeait la pédagogie universitaire : la transmission des savoirs. In Numérique et éducation : dispositifs, jeux, enjeux, hors-jeux sous la direction de Bonfils Ph., Dumas Ph. \& Massou L. - collection « Série Actes » de la revue Questions de communication (PUNEditions Universitaires de Lorraine), 125-144.

Charliet, B., Deschryver, N. \& Peraya, D. (2006). Apprendre en présence et à distance : une définition des dispositifs hybrides. Distances et savoirs, 2006/4 vol. 4, pp. 469-496.

Cohard, P. (2015). L'apprentissage dans les serious games : proposition d'une typologie. @GRH, 16(3), 11-40. doi :10.3917/grh.153.0011.

Conseil de l'Europe (2018). Cadre Européen Commun de Référence pour les Langues : apprendre, enseigner, évaluer. Volume complémentaire avec de nouveaux descripteurs. Strasbourg.

Conseil de l'Europe (2001). Cadre Européen Commun de Référence pour les Langues : apprendre, enseigner, évaluer. Strasbourg.

Cros, F. (2001). L'innovation scolaire. Paris : INRP.

Depover, C., De Lièvre, B., Peraya, D., Quintin, J. \& Jaillet, A. (2011). Le tutorat en formation à distance. Louvain-la-Neuve, Belgique : De Boeck Supérieur. doi :10.3917/dbu.depov.2011.01.

Develotte, C. (2010). Réflexions sur les changements induits par le numérique dans l'enseignement et l'apprentissage des langues. Études de linguistique appliquée, 2010/4 n 160, 445-464.

Deouël, J. \& Granjon, F. (éds.) (2011). Communiquer à l'ère numérique. Regards croisés sur la sociologie des usages. Paris : Presses des Mines.

Fourgous, J.-M. (2010). Sur la modernisation de l'école par le numérique. Réussir l'école numérique. Rapport de Mission parlementaire. 15 février 2010.

Grassin, J.-F. (2016). Échanges en ligne sur un réseau social dans le cadre d'une formation en Français Langue Étrangère : pratiques discursives et modes de participation. In Liénard, F. \& Zlitni, S. (éd.), Médias numériques et communication électronique, Actes de colloque, Bruges, 295-303. Guichon, N., Koné, S. \& Grassin, J.-F. (2017). The digital transition of international students. Canadian Association of Applied Linguistics, Canadian Association of Applied Linguistics, May 2017, Toronto, Canada. 〈hal-01533410〉

Martin, G. (2012). Les amis de vos amis sont-ils vos amis ? Idées économiques et sociales, 169(3), 1-1. doi :10.3917/idee.169.0001.

Moscovici, S. (1977). Essai sur l'histoire humaine de la nature. Paris : Flammarion.

Soubrié, T. (2014). Des enseignants en activité travaillent de concert avec des enseignants novices pour élaborer des scénarios pédagogiques : quelles sont leurs motivations ? Cahiers de l'ASDIFLE, 108-121.

Mangenot, F., Soubrié, T. (2014). Le web social au service de tâches d'écriture. Recherches, 89-105. Narcy-Combes, J. P. (2005). Didactique des langues et TIC : vers une recherche-action responsable. Paris : Ophrys.

Nissen, E. (2011). Variations autour de la tâche dans l'enseignement / apprentissage des langues aujourd'hui. Alsic [En ligne], vol. 14 | 2011, mis en ligne le 11 octobre 2011, Consulté le 02 mai 2019. DOI : $10.4000 /$ alsic.2344 
Nissen, E. (2004). Importance du scénario pédagogique dans l'apprentissage d'une langue étrangère en ligne. Les Langues modernes 4/2004, 14-24.

Nissen, E. (2006). Scénarios de communication en ligne dans des formations hybrides. Le Français dans le monde. Recherches et applications, « Les échanges en ligne dans l'apprentissage et la formation », numéro spécial, juillet 2006, 44-58.

Nissen, E. (2007). Quelles aides les formations hybrides en langues proposent-elles à l'apprenant pour favoriser son autonomie? Apprentissage des langues et systèmes d'information et de communication (ALSIC), vol. 10, $\mathrm{n}^{\circ} 1.129-144$.

Peraya, D., Bonfils, P. (2014). Détournements d'usages et nouvelles pratiques numériques : l'expérience des étudiants d'Ingémédia à l'Université de Toulon. Revue des sciences et techniques de l'information et de la communication pour l'éducation et la formation, 21.

Pomian, J. (2009). « Serious games » : apprendre en jouant ?. Quaderni, n 69, 121-126.

Sander, D. (2014). Traité de psychologie des émotions : Traité de psychologie des émotions. Paris : Dunod. Sauvage J. \& Billières, M. (éds.) (2019). « Enseigner la phonétique d'une langue étrangère. Bilan et perspectives. » Recherches en Didactique des Langues et des Cultures, 16-1. https:// journals.openedition.org/rdlc/4219

Sauvage, J. (2019a). Mobilité et appropriation des langues. Réponse à D. Meunier. Notion en Question : les mobilités. Recherches en didactique des langues et des cultures. Dans ce numéro

Sauvage, J. (2019b). Phonétique et didactique. Un mariage contre-nature. Recherches en didactique des langues et des cultures http://journals.openedition.org/rdlc/4276.

Sauvage, J., Dufour, S. \& Auger, N. (2017). Face à l'hétérogénéité des projets de mobilité Erasmus : Evaluer les enseignements de FLE de l'université Paul-Valéry - Montpellier 3. Le Langage et l'Homme, n' 52-1, 77-92.

Sauvage, J. (2017). Discours enseignant, dialogisme et idéologies académiques, Recherches en didactique des langues et des cultures [En ligne], 14-2 | 2017, mis en ligne le 15 juin 2017, URL : http://rdlc.revues.org/1869; DOI : 10.4000/rdlc.1869

Soubrié, T. (2016). La pratique réflexive, une dimension centrale dans la formation des enseignants à l'intégration du numérique dans l'éducation. In : Christian Ollivier, Thierry Gaillat, Laurent Puren (dir.). Numérique et formation des enseignants de langue. Pistes et imaginaires, Editions des archives contemporaines, pp. 1-18. 〈hal-01654292〉

Tremblay, N. (1986). Apprendre en situation d'autodidaxie. Montréal : Presses de l'université de Montréal.

Weber, C. (2019). Interrogations épistémologiques autour de l'oralité ", Recherches en didactique des langues et des cultures [En ligne], 16-1 | 2019, mis en ligne le 30 janvier 2019, consulté le 02 mai 2019. URL : http://journals.openedition.org/rdlc/4252.

\section{RÉSUMÉS}

Le présent article a pour objectif d'aborder les difficultés inhérentes à la création d'une formation innovante dans l'enseignement d'une langue étrangère. En l'occurrence, il s'agit de réfléchir à propos de l'élaboration d'un diplôme d'université de FLE organisé de manière hybride, c'est-à-dire en s'appuyant sur les dispositifs numériques permettant la mise en place de séquences d'apprentissage en autonomie et à distance. Nous insisterons notamment sur la 
nécessité de ne pas galvauder le terme d'innovation, et nous verrons qu'une pédagogie qui se veut réellement innovante nécessite une réflexion de fond tant sur le plan pédagogique que sur les plans administratif et technologique pour répondre aux besoins de mobilité actuelle des étudiants internationaux.

The aim of this paper is to address the challenges of creating innovative training in foreign language teaching. In this case, we focus on developing a hybrid French as a Foreign Language university diploma, relying on digital devices allowing for the implementation of learning sequences accessible to the distance learner. We will insist in particular on the need to avoid misusing the term of innovation, and we will see that a pedagogy which wants to be really innovative requires a fundamental reflection not only on the pedagogic level but also on the administrative and technological fronts to be able to answer to international students' needs regarding their mobilities.

\section{INDEX}

Mots-clés : apprentissage en autonomie ; innovation ; didactique du FLE ; formation hybride ; ingénierie pédagogique

Keywords : self-learning; innovation; learning and teaching in french as a foreign language; hybrid training; educational engineering

\section{AUTEUR}

\section{JÉRÉMI SAUVAGE}

Université Paul Valéry Montpellier 3, CNRS, PRAXILING UMR 5267, F34000, Montpellier Jérémi Sauvage est Maître de conférences habilité à diriger des recherches en Sciences du langage à l'université Paul-Valéry - Montpellier 3, membre du laboratoire Praxiling UMR 5267 CNRS. Ses domaines de recherche sont l'acquisition et la didactique des langues, plus particulièrement en phonétique / phonologie.

jeremi.sauvage[at]univ-montp3.fr 